\title{
Efecto de la estacionalidad sobre el estado nutricional de Hierro, Zinc y Cobre
}

\author{
Effect of seasonality on nutritional status \\ of Iron, Zinc and Copper
}

\begin{abstract}
Food consumption and therefore the nutritional status of micronutrients are influenced by various factors such as seasonality. This study aimed to determine the effect of seasonality on the nutritional status of $\mathrm{Fe}, \mathrm{Zn}$ and $\mathrm{Cu}$ in adult subjects. A longitudinal study was conducted during the months of September 2005 and August 2006. Fifty subjects of both sexes belonging to the urban area of the city of Santiago de Chile were evaluated. Blood samples were drawn during the first week of each month; serum iron, copper and zinc, hemoglobin, mean cell volume, serum ferritin, zinc-protophorphyrin and ceruloplasmin were determined. Serum copper and zinc levels showed significant seasonal variations but not serum iron. Mean cell volume, hemoglobin and ceruloplasmins were significantly lower during spring and summer while serum ferritin and zinc-protophorphyrin showed lower concentrations during autumn and winter. We conclude that during the spring and summer levels of serum iron, copper and zinc could be diminished by reduced food intake.

Key words: Iron, copper, zinc, seasonality, nutrition status.
\end{abstract}

\section{INTRODUCCIÓN}

Los micronutrientes hierro (Fe), zinc $(\mathrm{Zn})$ y cobre $(\mathrm{Cu})$ son esenciales para la vida pues forman parte de proteínas y enzimas que actúan en diversos procesos biológicos indispensables para el funcionamiento de un organismo vivo (1). El hombre, requiere absorberlos desde sus alimentos ingeridos durante toda la vida especialmente en etapas de rápido crecimiento. Sin embargo, las dietas de la mayoría de las poblaciones son frecuentemente deficientes en estos microminerales.

La deficiencia de Fe continúa siendo la carencia nutricional más común en el mundo, afectando sobre todo a niños, mujeres en edad fértil y embarazadas (2). Se estima un déficit de $\mathrm{Zn}$ en igual proporción sobre todo en países en desarrollo, pero es poco reconocido debido a la falta de biomarcadores sensibles (1). El déficit de Cu es menos frecuente y se encuentra en grupos específicos, tales como niños con desnutrición o individuos con enfermedades que impliquen trastornos de la absorción (3). Un hecho común entre estos tres micronutrientes es el bajo aporte en la dieta del metal en forma biodisponible.

Para evaluar estado de nutrición de estos minerales se utiliza diferentes biomarcadores. De los tres, la evaluación nutricional de Fe es la que presenta menos problemas, pues cuenta con biomarcadores más eficientes (1). Para la evaluación
Melissa Miranda D. Manuel Olivares G. Magdalena Araya Q. Fernando Pizarro A.

Laboratorio de Micronutrientes, Instituto de Nutrición y Tecnología de los Alimentos INTA, Universidad de Chile. Santiago, Chile.

Dirigir la correspondencia a:
Profesor
Fernando Pizarro A.
Laboratorio de Micronutrientes
Instituto de Nutrición y Tecnología de los Alimentos (INTA)
Universidad de Chile
Av. El Líbano 5524, Macul, Santiago, Chile
Teléfono: +5629781551
Fax: +5622214030
E-mail: fpizarro@inta.uchile.cl

Este trabajo fue recibido el 4 de Diciembre de 2012 y aceptado para ser publicado el 7 de Mayo de 2013.

del estado nutricional de Zn existe gran número de propuestas pero todas presentan problemas que afectan su validez, las pruebas más comunes usan índices bioquímicos estáticos basadas en la concentración de Zn en tejidos, siendo el más utilizado la medición de Zn sérico o plasmático (4). Cobre sérico y ceruloplasmina son los indicadores frecuentemente usados para evaluar el estado nutricional de cobre, se ha tratado de definir otros biomarcadores pero estos sólo son eficientes y útiles cuando la exposición es lo suficientemente alta como para inducir manifestaciones clínicas (5).

El estado de nutrición de los micronutrientes en general depende de la ingesta de alimentos, y las concentraciones de estos en los alimentos varía dependiendo de las características innatas de las plantas o animales (variedades, madurez, genética y edad), factores ambientales (suelo, ubicación geográfica, fuente de agua, uso de fertilizantes y estación), factores culturales, económicos, sociales, mercadeo, además de los métodos de manipulación procesamiento y cocción. Sin embargo, la estación del año puede llegar a tener una gran influencia en la ingesta de nutrientes, pudiendo ser marcada en el caso de los micronutrientes $(6,7)$. En Chile, no existen estudios que analicen esta variable y que aporten antecedentes sobre este tema. El presente estudio tuvo por objetivo determinar el efecto de la estacionalidad sobre el estado nutricional de hierro, zinc y cobre de sujetos adultos. 


\section{SUJETOS Y METODOS}

Sujetos y diseño del estudio

Un estudio longitudinal prospectivo fue conducido entre los meses de septiembre del 2005 y agosto del 2006. Se evaluaron 50 sujetos aparentemente sanos (54\% mujeres) de ambos sexos pertenecientes a la zona urbana de la ciudad de Santiago de Chile, de nivel socioeconómico medio bajo y entre las edades de 18 a 62 años. Los participantes mantuvieron sus hábitos de vida a lo largo del estudio, ninguno tomaba suplementos nutricionales, ni medicamentos incluidos anticonceptivos orales. Los sujetos fueron citados en ayunas a una toma de sangre mensual (primera semana de cada mes) en días consecutivos (de lunes a miércoles). Además, se realizó una encuesta detallada de salud que incluyó historia de patologías y estado de salud actual.

\section{Ética}

Se obtuvo un consentimiento informado de todos los voluntarios antes del inicio del estudio. Los sujetos recibieron información detallada acerca del protocolo, la participación fue voluntaria, no hubo ningún tipo de remuneración y fueron libres de abandonar el estudio en cualquier momento.

El protocolo fue aprobado por el Comité de Ética del Instituto de Nutrición y Tecnología de Alimentos (INTA) de la Universidad de Chile antes de su ejecución.

Determinaciones bioquímicas y hematológicas

En cada mes y después de un ayuno nocturno, se extrajo una muestra de sangre de $10 \mathrm{ml}$ por punción venosa cubital entre las 8:00 y las 9:00 hrs. para la determinación de hemoglobina $(\mathrm{Hb})$ y volumen corpuscular medio (VCM) (CELL-DYN 1700, ABBOTT Diagnostics, Abbott Park, IL); protoporfirina libre eritrocitaria (Zpp) (ZP Hematofluorometer Model 206D, AVIV Biomedical Inc., Lakewood, NJ); ferritina sérica por ELISA (FS) (8); las concentraciones de Fe, Cu y Zn séricos por espectrofotometría de absorción atómica con horno de grafito (Perkin Elmer, Model SIMAA 6100, The Perkin-Elmer Corporation, Norwalk, CT, USA) y ceruloplasmina (Cp) se midió por nefelometría (sistema de matriz de proteína; Beckman Instruments Inc., Brea, CA, EE.UU).

La anemia se definió como una $\mathrm{Hb}$ por debajo del punto de corte de referencia ( $\mathrm{Hb}<12 \mathrm{~g} / \mathrm{dL}$ en mujeres y $<13 \mathrm{~g} / \mathrm{dL}$ en hombres) (8); anemia por deficiencia de hierro, como una $\mathrm{Hb}$ por debajo del punto de corte de referencia más dos de los indicadores de laboratorio de estado nutricional de hierro alterados (VCM<80 fL y/o ZPP>70 ug/dL GR y/o FS<12 ug/L) (9). Los depósitos de hierro depletados como, FS por debajo del punto de corte de referencia (FS $<12 \mathrm{ug} / \mathrm{dL}$ ) (8). Los niveles deficientes de Cu se definieron como valores séricos $<80$ $\mu \mathrm{g} / \mathrm{dl}$ en mujeres $\mathrm{y}<70 \mu \mathrm{g} / \mathrm{dl}$ en hombres (10) y los niveles deficientes en zinc como valores séricos $<70 \mu \mathrm{g} / \mathrm{dl}$ en mujeres $y<74 \mu \mathrm{g} / \mathrm{dl}$ en hombres (11).

\section{Análisis estadístico}

Para el análisis de la información se utilizó el programa SPSS versión 19. La normalidad de las variables fue examinada mediante la prueba de Shapiro-Wilk. Las variables que mostraron distribución gaussiana se presentan en promedio y desviación estándar. La variable FS fue normalizada mediante la transformación a su logaritmo natural debido a que presentó una distribución asimétrica (12). El efecto de la estacionalidad sobre los niveles séricos de $\mathrm{Fe}, \mathrm{Cu}$ y $\mathrm{Zn}$ y los diferentes indicadores evaluados fue determinado por análisis de varianza (ANOVA) de dos vías para muestras repetidas, con sexo y tiempo como variables y ANOVA de una vía y prueba de post-hoc de Bonferroni. Para determinar la influencia de la estación en los distintos parámetros evaluados se definió las estaciones de la siguiente forma: primavera (octubre, noviembre, diciembre), verano (enero, febrero, marzo), otoño (abril, mayo, junio), Invierno (julio, agosto, septiembre). Valores $\mathrm{P}$ $<0,05$ fueron considerados significativos.

\section{RESULTADOS}

Del total de sujetos fueron eliminados 2 por tener menos de 8 evaluaciones. Los sujetos analizados ( 22 hombres y 26 mujeres) tuvieron un promedio de edad de $43 \pm 12$ y $40 \pm 12$ años para hombres y mujeres respectivamente.

Como se aprecia en el gráfico 1, las concentraciones de Fe y Cu sérico variaron significativamente por sexo durante los diferentes meses de estudio (ANOVA de dos vías para muestras repetidas, $p<0,05)$ mientras que el promedio de $Z n$ sérico no presento diferencias significativas entre sexos. Los niveles de Fe se mantuvieron superiores en los hombres, mientras que en las mujeres los niveles de Cu fueron mayores durante todos los meses de evaluación.

Cobre y Zn séricos presentaron variaciones estacionales significativas (ANOVA de dos vías para muestras repetidas, $\mathrm{p}<0,05)$, pero no así Fe, el cual permaneció constante durante todo el estudio (gráfico 1). En el caso de Cu sérico, se observó una variación significativa entre los meses de primavera e invierno, verano y otoño y verano invierno ( $p<0,05$ post-hoc de Bonferroni). En relación a los niveles de $\mathrm{Zn}$, los meses primavera y verano, primavera y otoño y primavera e invierno variaron significativamente ( $p<0,05$ post-hoc de Bonferroni).

En la tabla 1 se puede observar que todos los indicadores bioquímicos evaluados tuvieron una variación estacional significativa (ANOVA de dos vías para muestras repetidas, $p<0,05$ ). Los promedios de VCM, Hb y Cp fueron significativamente menores durante primavera y verano en comparación con otras estaciones. Distintas tendencias estacionales se observaron para FS y Zpp que presentaron las concentraciones más bajas durante otoño e invierno.

En relación a las diferencias por sexo, no se observaron significancias a lo largo de la evaluación para VCM, mientras que durante todos los meses de evaluación los niveles $\mathrm{Hb}$ y FS fueron significativamente menores en las mujeres. Por otro lado, las concentraciones de Zpp y Cp fueron significativamente mayores para las mujeres en relación a los hombres (ANOVA de dos vías para muestras repetidas, $\mathrm{p}<0,05$ ).

\section{DISCUSIÓN}

El consumo de alimentos y por lo tanto el estado de nutrición de los nutrientes está condicionado por diferentes factores entre los que se cuenta la estacionalidad $(6,7)$. En Chile no se cuenta con estudios que evalúen este aspecto y menos aún en micronutrientes como $\mathrm{Fe}$, $\mathrm{Cu}$ y $\mathrm{Zn}$ que pueden verse mayormente afectado por cambios en el consumo de alimentos. En este estudio se evaluó el efecto de la estacionalidad sobre el estado nutricional de hierro, zinc y cobre de sujetos adultos, observándose diferencias significativas por sexo en las concentraciones de $\mathrm{Fe}$ y $\mathrm{Cu}$ y diferencias estacionales en $\mathrm{Cu}$ y $\mathrm{Zn}$.

El cobre sérico y la ceruloplasmina son los indicadores frecuentemente usados para evaluar el estado nutricional de cobre. Las diferencias significativas por sexo observadas para Cu y Cp en este estudio están bien documentadas, y son consecuencia de las variaciones del ciclo estrógenos progesterona en las mujeres; el alto nivel de estrógenos estimula la síntesis 
de ceruloplasmina y aumenta los niveles plasmáticos de este mineral, actuando como factor protector (13). Con respecto a las variaciones en el tiempo, en condiciones normales, la concentración de cobre en el plasma es aparentemente regulada por sólidos mecanismos homeostáticos. Personas saludables de vida libre y personas que consumen dietas con un adecuado contenido de cobre mantienen sus concentraciones plasmáticas de cobre dentro de un rango de $\pm 6.7-8.6 \%$ durante períodos de hasta 13 meses $(14,15)$. Sin embargo, las concentraciones plasmáticas de cobre son más variables ( \pm 20\%) cuando los sujetos se alimentan con una dieta baja en cobre (14). Por lo tanto las bajas concentraciones observadas de Cu y Cp durante primavera y verano pueden deberse a una disminución en la ingesta de alimentos ricos en este nutriente como carnes y derivados, frutos secos y legumbres $(7,16-18)$.

Por otro lado, las diferencias por sexo observadas en las concentraciones de Fe sérico, $\mathrm{Hb}$ y FS son las esperadas puesto que la normal distribución de estos indicadores es dependiente de la edad y del sexo. La homogeneidad en las concentraciones de Fe sérico, hemoglobina, VCM, FS y la ausencia de anemia por deficiencia de hierro pueden deberse a que hoy en día existe una mayor disponibilidad en todas las estaciones de vegetales de hoja verde y otros alimentos ricos en nutrientes formadores de células sanguíneas. Además, actualmente en Chile por ley la harina de trigo se encuentra fortificada con hierro (30 mg de Fe como sulfato ferroso/Kg) y vitaminas del complejo B (19), esta intervención ha contribuido fuertemente a la disminución de anemia por deficiencia de hierro en Chile (20) y según se observa en este estudio estos efectos positivos no excluyen a los adultos. Sin embargo la disminución significativa observada en los niveles de Hb y VCM durante los meses de primavera y verano puede deberse a una menor ingesta de alimentos ricos en $\mathrm{Fe}$, al igual que en el caso de cobre, pues comparten similares fuentes alimenticias $(6,7,16-18)$.

\section{FIGURA 1}

Efecto de la estacionalidad sobre los niveles séricos de $\mathrm{Fe}$, Cu y Zn por sexo.

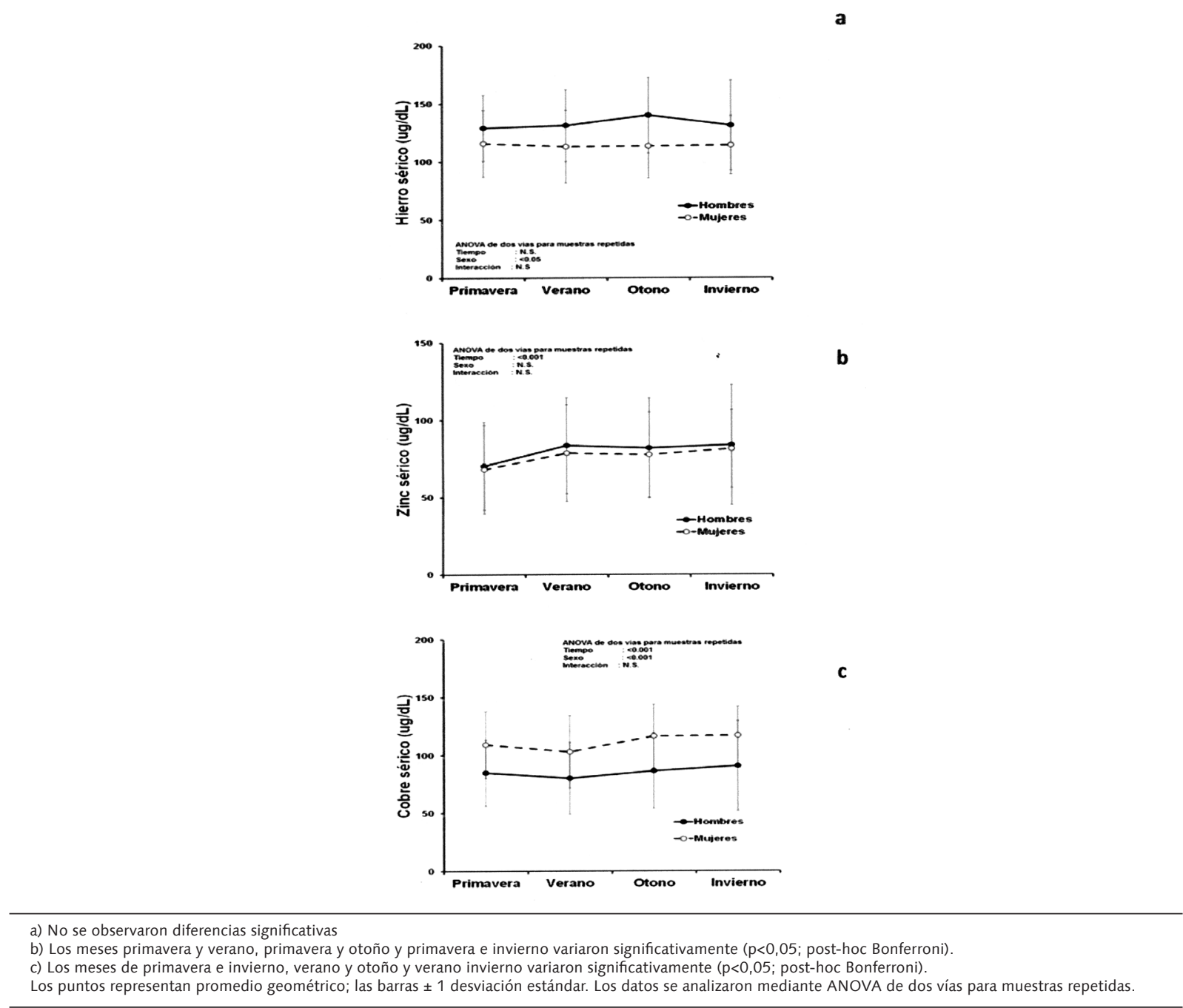


El déficit de zinc usualmente está relacionado con el bajo consumo de alimentos ricos en este mineral. Según se demostró en este estudio, hubo una variación significativa de los niveles de $\mathrm{Zn}$ durante los diferentes meses y en especial durante primavera se observó una caída importante en el promedio tanto para hombres como mujeres, encontrándose por debajo del punto de corte considerado como normal. En un estudio realizado en una muestra representativa del Gran Santiago, donde se determinó la ingesta de $\mathrm{Fe}$, $\mathrm{Cu}$ y $\mathrm{Zn}$ de los alimentos más consumidos se observó que altos porcentajes de la población, de todos los grupos etarios, presentaban ingestas de zinc por debajo del Requerimiento Promedio Estimado (21). Además, sumado a esto la falta de buenos indicadores que permitan establecer con certeza la deficiencia de zinc, se cree que más sujetos de este estudio pueden estar deficitarios en zinc especialmente en los meses de primavera y verano debido al menor consumo de alimentos ricos en Zn como carnes rojas, mariscos, hígado entre otros, ya que por el calor el consumo de estos puede estar disminuido $(7,16-18)$.

Finalmente, creemos que este estudio tiene la limitación de no contar con la evaluación del consumo alimentario de los sujetos evaluados y no haber registrado la morbilidad, ya que es sabido que los procesos infecciosos o inflamatorios modifican los indicadores de laboratorio de estatus de hierro, zinc y cobre. Finalmente, no podemos descartar que algunas variaciones fisiológicas pudieran haber afectado el metabolismo de estos microminerales, tales como cambios hormonales dependientes de la exposición lumínica o variaciones en el volumen plasmático secundarios a variaciones en la temperatura ambiental promedio. Concluimos, que aun cuando habrían otras posibles causas que justifiquen los cambios, la estacionalidad es un factor a considerar cuando se realicen mediciones de estado de nutrición de los microminerales hierro, zinc y cobre.

\section{RESUMEN}

El consumo de alimentos y por lo tanto el estado de nutrición de los micronutrientes está condicionado por di- ferentes factores como la estacionalidad. El presente estudio tuvo como objetivo determinar el efecto de la estacionalidad sobre el estado nutricional de hierro, zinc y cobre de sujetos adultos habitantes de la ciudad de Santiago de Chile. Un estudio longitudinal en 50 sujetos de ambos sexos se realizó entre los meses de septiembre del 2005 y agosto del 2006. Durante la primera semana de cada mes se extrajo muestras de sangre para la determinación de hierro, zinc y cobre sérico, hemoglobina, volumen corpuscular medio, ferritina sérica zincprotoporfirina y ceruloplasmina. Los niveles de cobre, zinc, hemoglobina, volumen corpuscular medio, y ceruloplasmina fueron significativamente menores durante primavera y verano mientras ferritina sérica y zinc-protoporfirina presentaron niveles más bajas durante otoño e invierno. Concluimos que la estacionalidad es un factor a considerar cuando se realicen mediciones de estado de nutrición de los microminerales hierro, zinc y cobre.

Palabras clave: Hierro, cobre, zinc, estacionalidad, estado de nutrición.

\section{BIBLIOGRAFÍA}

1. Hambidge M. Biomarkers of trace minerals intake and status. J Nutr 2003; 133: 948S-55S.

2. International Nutritional Anemia Consultative Group (INACG). Measurement of iron status, The Nutrition Foundation, Washington DC. 1985.

3. Olivares $M$, Hertrampf $E$, Uauy R. Copper and Zinc interactions in anemia: a public health perspective. En: Nutritional Anemia. Kraemer K, Zimmermann MB., eds. Sight and Life Press, Basel, Swisszerland; 2007: p. 99-109.

4. Ruz M, Cavan KR, Bettger WJ, Thompson L, Berry M, Gibson RS. Development of a dietary model for the study of mild zinc deficiency in humans and evaluation of some biochemical and functional indices of zinc status. Am J Clin Nutr. 1991; 53: 1295-303.

5. Araya $M$, Pizarro F, Olivares $M$, Arredondo $M$, Gonzales $M$, Méndez $M$. Understanding copper homeostasis in humans and copper effects on health. Biol Res 2006; 39: 183-87.

TABLA 1

Variaciones estacionales en las concentraciones de volumen corpuscular medio, hemoglobina, ferritina sérica, zinc protoporfirina, Ceruloplasmina por sexo (promedio $\pm \mathrm{DE}$ )

\begin{tabular}{|c|c|c|c|c|c|c|c|c|c|c|c|}
\hline & \multicolumn{2}{|c|}{$\begin{array}{l}\text { Primavera } \\
(\mathrm{n}=50)\end{array}$} & \multicolumn{2}{|c|}{$\begin{array}{l}\text { Verano } \\
(n=50)\end{array}$} & \multicolumn{2}{|c|}{$\begin{array}{l}\text { Otoño } \\
(n=48)\end{array}$} & \multicolumn{2}{|c|}{$\begin{array}{l}\text { Invierno } \\
(n=48)\end{array}$} & \multicolumn{3}{|c|}{$\begin{array}{l}\text { ANOVA de dos vías para } \\
\text { muestras repetidas }\end{array}$} \\
\hline & $\mathrm{H}$ & $M$ & $\mathrm{H}$ & $M$ & $\mathrm{H}$ & $M$ & $\mathrm{H}$ & $M$ & Tiempo & Sexo & Interacción \\
\hline VCM (fL) & $89 \pm 2^{a}$ & $88 \pm 3^{a}$ & $89 \pm 2^{a}$ & $88 \pm 3^{a}$ & $90 \pm 2^{b}$ & $89 \pm 3^{b}$ & $90 \pm 2^{b}$ & $89 \pm 3^{b}$ & $<0.001$ & 0.271 & 0.386 \\
\hline $\mathrm{Hb}(\mathrm{g} / \mathrm{dL})$ & $15 \pm 1^{a}$ & $14 \pm 1^{a}$ & $15 \pm 1^{a}$ & $13 \pm 1^{a}$ & $16 \pm 1^{b}$ & $14 \pm 1^{b}$ & $16 \pm 1^{b}$ & $14 \pm 1^{b}$ & $<0.001$ & $<0.001$ & 0.366 \\
\hline FS (ug/L)* & $\begin{array}{c}57^{a} \\
(35-95)\end{array}$ & $\begin{array}{c}28^{a} \\
(15-51)\end{array}$ & $\begin{array}{c}62^{b} \\
(36-107)\end{array}$ & $\begin{array}{c}32^{\mathrm{b}} \\
(18-57)\end{array}$ & $\begin{array}{c}61^{a} \\
(36-102)\end{array}$ & $\begin{array}{c}27^{a} \\
(15-50)\end{array}$ & $\begin{array}{c}53^{\mathrm{a}} \\
(31-91)\end{array}$ & $\begin{array}{c}25^{\mathrm{a}} \\
(13-48)\end{array}$ & $<0.001$ & $<0.001$ & 0.141 \\
\hline $\begin{array}{l}\text { Zpp } \\
\text { (ug/dL GR) }\end{array}$ & $58 \pm 10^{a}$ & $64 \pm 11^{a}$ & $50 \pm 8^{b}$ & $55 \pm 11^{b}$ & $34 \pm 6^{c}$ & $42 \pm 10^{c}$ & $44 \pm 10^{d}$ & $50 \pm 15^{d}$ & $<0.001$ & $<0.05$ & 0.370 \\
\hline $\mathrm{Cp}(\mathrm{mg} / \mathrm{L})$ & $22 \pm 10^{a}$ & $31 \pm 11^{a}$ & $21 \pm 9^{a}$ & $27 \pm 11^{a}$ & $24 \pm 9^{b}$ & $32 \pm 11^{b}$ & $26 \pm 11^{b}$ & $33 \pm 11^{b}$ & $<0.001$ & $<0.05$ & 0.137 \\
\hline
\end{tabular}

*Promedio geométrico y rango \pm 1 desviación estándar

Valores con letras diferentes en una fila difieren significativamente $(p<0,05$ ANOVA con test de Bonferroni)

H, Hombres; M, Mujeres; VCM, Volumen Corpuscular Medio; Hb, Hemoglobina; FS, Ferritina Sérica; Zpp, Zinc protoporfirina; Cp, Ceruloplasmina. 
6. Ronnenber Alayne G, Goldman Marlene, Aitken Iain, $\mathrm{Xu}$ Xiping. Anemia and Deficiencies of Folate and Vitamin B-6 Are Common and Vary with Season in Chinese Women of Childbearing Age J Nutr 2000; 130: 2703-10.

7. Tianan Jiang, Parul Christian, Subarna K. Khatry, Lee Wu, Keith P. West. Micronutrient Deficiencies in Early Pregnancy Are Common, Concurrent, and Vary by Season among Rural Nepali Pregnant Women. J Nutr 2005; 135: 1106-12.

8. International Nutritional Anemia Consultative Group (INACG). Measurements of Iron Status. The Nutrition Foundation, Washington DC; 1985.

9. Gleason G, Scrimshaw N. An overview of the functional significance of iron deficiency. Nutritional Anemia. Kraemer K, Zimmermann MB., eds. Sight and Life Press, Basel, Swisszerland; 2007: p 45-57.

10. Elin RJ. Reference intervals and laboratory values. En: Cecil Textbook of Medicine. 22th ed. Goldman L, Ansiello DA, eds. WB Saunders, Philadelphia, PA; 2004: 2496-2505.

11. International Zinc Nutrition Consultative Group (IZiNCG). Assessment of the risk of zinc deficiency in populations and options for its control. Hotz C, Brown KH, eds. Food Nutr Bull 2004; 25: S91-S204.

12. Taucher E. Bioestadística, segunda edición, Editorial Universitaria. Santiago, Chile; 1997.

13. Ayala M, Pizarro F, Méndez M, Arredondo, M, Araya M. Copper and liver function indicators vary depending of the female hormonal cycle and serum hormonal binding globulin concentration en healthy women, Biol Trace Elem Res. 2008; 121:9-15

14. Milne DB, Johnson PE, Klevay LM, Sandstead HH. Effect of copper intake on balance, absorption and status indices of copper in men. Nutr Res. 1990; 10: 945-86.

15. Gallagher SK, Johnson LK, Milne DB. Short-term and longterm variability of indices related to nutritional status. I. $\mathrm{Ca}, \mathrm{Cu}, \mathrm{Fe}, \mathrm{Mg}$, and Zn. Clin Chem. 1989; 35: 369-73.

16. Tarasuk Valerie, Beaton George. The nature and individuality of within-subject variation in energy intake. Am J Clin Nutr. 199 1; 54: 464-70.

17. Durán F Eliana, Soto A Delia, Labraña T Ana María, Pradenas $P$ Francisco. Estacionalidad y días de la semana como factores de riesgo de la dieta de mujeres adultas. Rev Chil Nutr. 2005; 32: 254-61.

18. Basiotis Peter, Thomas Robin, Kelsay June, Mertz Walter. Sources of variation in energy intake by men and women as determined from one year's daily dietary records. Am J Clin Nutr. 1989; 50: 448-53.

19. Peña G, Pizarro F, Hertrampf E. Aporte del hierro del pan a la dieta chilena. Rev Med Chil. 1991; 119: 753-57.

20. Olivares M, Pizarro F, Hertrampf E, Walter T, Arredondo M, Letelier A. Fortificación de Alimentos con hierro en Chile. Rev Chil Nutr. 2000; 27:340-4.

21. Olivares M, Pizarro F, de Pablo S, Araya M, Uauy R. Iron, zinc and copper contents in common Chilean foods and daily intakes in Santiago City, Chile. Nutrition 2004; 20: 205-12. 